\title{
Single-Sweep Pulmonary Vein Isolation using the new third-generation laser balloon - Evolution in ablation style using endoscopic ablation system
}

\author{
Shota Tohoku ${ }^{1}$, Stefano Bordignon ${ }^{1}$, Luca Trolese ${ }^{1}$, Simone Zanchi ${ }^{1}$, Lorenzo Bianchini ${ }^{1}$, \\ Felix Operhalski ${ }^{2}$, Shaojie Chen ${ }^{3}$, KR Julian Chun ${ }^{1}$, and Boris Schmidt ${ }^{1}$ \\ ${ }^{1}$ Cardioangiologisches Centrum Bethanien \\ ${ }^{2}$ Universitätsklinikum Frankfurt \\ ${ }^{3}$ Agaplesion Markus Krankenhaus
}

May 28, 2021

\begin{abstract}
Background: The endoscopic ablation system (EAS) is an established ablation device for pulmonary vein isolation (PVI) in patients with atrial fibrillation (AF). The novel X3 EAS is now equipped with a contiguous circumferential ablation mode (RAPID mode). Aim: To determine the feasibility of single-sweep ablation using X3. Methods: Consecutive patients who underwent AF ablation using X3 were enrolled. We assessed the acute procedural data focusing on "Single-sweep PVI" defined as PVI with a single energy application using RAPID mode to complete the circular lesion set, and on "first-pass isolation" defined as successful visually guided PVI after initial circular lesion set. Results: One-hundred AF patients (56\% male, age $68 \pm 10$ years, $66 \%$ paroxysmal AF) were analyzed. A total of 379 of 383 PVs (99\%) were isolated with X3. Single-sweep isolation and first-pass-isolation were achieved in 214 PVs (56\%) and in 362 PVs (95\%), respectively. Single-sweep isolation rates varied across PVs with higher rates at the superior PVs (61.2\% vs. inferior PVs:49.5\%, P=0.0239) and at PVs with maximal ostial diameter $<24 \mathrm{~mm}(57.6 \%$ vs. $>24 \mathrm{~mm}: 36.8 \%, \mathrm{P}=0.0151)$. The mean total procedure and fluoroscopy times were $43.0 \pm 10$ and $4.0 \pm 2 \mathrm{mins}$, respectively. In none of the patients an acute thromboembolic event (stroke or transient ischemic attack) or a pericardial effusion/tamponade occurred. A single transient phrenic nerve palsy was observed. Conclusion: The new X3 EAS allows for single-sweep PVI in 56\% of PVs. The new RAPID ablation mode leads to an improved rate of first-pass isolation associated with very short procedure times without compromising safety.
\end{abstract}

\section{Hosted file}

JCE X3_100_Manuscript_10.05.21.pdf available at https://authorea.com/users/336771/articles/ 524015-single-sweep-pulmonary-vein-isolation-using-the-new-third-generation-laserballoon-evolution-in-ablation-style-using-endoscopic-ablation-system 